\title{
Integrated Electrothermal Modeling of RF MEMS Switches for Improved Power Handling Capability
}

\author{
Brian D. Jensen ${ }^{1 *}$, Zhondge Wang ${ }^{2}$, Linda Chow ${ }^{1}$, Kazuhiro Saitou ${ }^{1}$, \\ Katsuo Kurabayashi ${ }^{\mathrm{P}}$, and John L. Volakis ${ }^{3}$ \\ 1. Department of Mechanical Engineering, University of Michigan, Ann Arbor,'MI \\ 2. Elec. Engineering and Computer Sci. Dept., University of Michigan, Ann Arbór, MI \\ 3. Department of Electrical Engineering, Ohio State University, Columbus, $\mathrm{OH}$ \\ * Presenting Author
}

RF MEMS switches have been presented by several researchers for use in transmit/receive switch circuits, tunable filters, and beam steering of antenna arrays [1], [2]. The benefits of RF MEMS switches are their low on-impedance, high off-impedance, highly, linear behavior, and, in many cases, nearly zero power consumption [3]. Drawbacks of RF MEMS switches include slow switching, high actuation voltage, high packaging cost, and low power handling capability [3]. Of these challenges, there appears to be substantial room to improve power handling without sacrificing device performance. Hence, this paper focuses on developing models predicting the power handling capability of a switch and corroborating these models with experiments. In particular, our model incorporates a new finite element-boundary integral method to solve for the electromagnetic fields.

Fig. 1 shows an explanatory drawing of a sample RF MEMS shunt switch. The switch consists of a beam placed between the ground lines of a CPW waveguide. Electrostatic force pulls the beam down into contact with the signal line, where metal-to-metal contact with the dimple shorts the waveguide. When the beam is down, current flows across the contact and through the beam, causing them to heat up. For high power, this joule heating

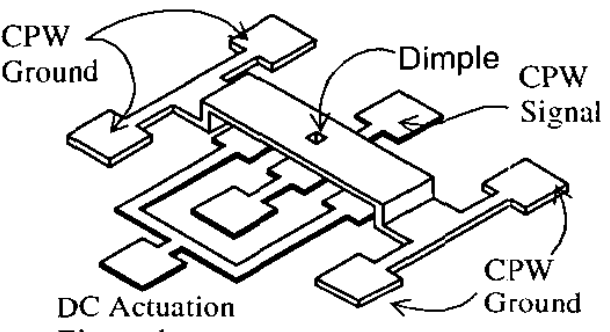
Electrode

Figure 1: Sample RF MEMS switch causes melting or welding of the contact, leading to failure. To accurately predict the power handling capability of a switch, we have developed a linked electrothermal switch model. This model predicts the current density using electromagnetic modeling, which is then used as a heat source in the thermal model, temperature giving a temperature prediction. Finally, a nanoscale model of the contact point predicts the temperature at the contact, which is the highest temperature in the switch.

\section{Multi-Physics Modeling}

The first step is simulation of the current density. We used a new formulation developed to simulate MEMS switches. This formulation simultaneously models a finite element domain and a detached boundary element domain in three dimensions [4]. This allows easy modeling of the substrate and the MEMS beam. The substrate is modeled using well-established finite element modeling, while the MEMS beam is modeled using boundary elements, allowing deformation of the beam without requiring continuous remeshing of the problem. Moreover, the required unknown-current density-is solved for directly in the boundary element domain. The model also includes the effect of the electrical contact resistance at the contact point. In this way, the current density throughout the switch and the contact is calculated with sufficient accuracy to predict local conductor losses. 
The conductor losses are then used as a heat generation source in the thermal model. Thermal effects are simulated using a two-dimensional finite element method which assumes constant temperature through the thickness of the beam. The model also includes the effect of thermal contact resistance at the contact point, restricting heat flow across the contact. In addition, because electrical resistivity of the metal is a strong function of temperature, the model iterates to find a convergent temperature solution. Convergence to within $1 \%$ on the Kelvin scale is normally achieved after two or three iterations.

When current flows through an electric contact, the maximum temperature in the device typically occurs at the individual contact asperities [5]. This asperity temperature is called the contact supertemperature, and it is a function of the temperature in the micromachined beam, which is calculated using the electromagnetic and thermal models described above. Using a nanoscale model of the microscopic contact asperities, the supertemperature is calculated, which represents the highest temperature in the switch and the point of initial switch failure. For example, contacts made of gold have been experimentally shown to soften and plastically deform at temperatures as low as $100^{\circ} \mathrm{C}$ [6]. Hence, failure is likely if the linked electro-thermal model, which includes the nanoscale supertemperature model, predicts a gold asperity temperature higher than this limit.

\section{Sample Results and Experimental Validation}

Fig. 2 shows a contour plot of the asperity supertemperature in a switch similar to the one shown in Fig. 1. The results show that supertemperature rises rapidly with both signal power and contact resistance. For contact resistance as low as $0.5 \Omega$, only about $200 \mathrm{~mW}$ of carried power will cause contact softening for this case.

In order to validate the model predictions, RF MEMS devices are now being fabricated with integrated temperature sensors to allow measurement of the beam temperature. The model will then

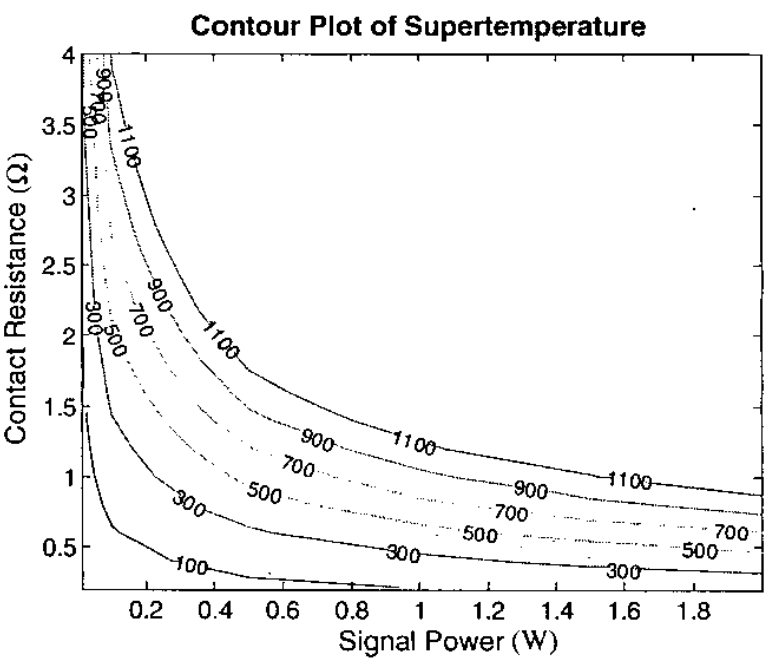

Figure 2: Contour plot of asperity supertemperature be used to design switches for improved power handling performance.

\section{References}

[1] C. T.-C. Nguyen. L. P. B. Katehi, and G. M. Rebeiz, "Micromachined devices for wireless communications," Proceedings of the IEEE, vol. 86, pp. 1756-1768, Aug. 1998.

[2] C. Goldsmith, J. Kleber, B. Pillans, D. Forehand, A. Malczewski, and P. Frueh, "RF MEMS: Benefits \& challenges of an evolving RF switch technology," in 200I IEEE GaAs Digest, pp. 147-148, 2001.

[3] G. M. Rebeiz and J. B. Muldavin, "RF MEMS switches and switch circuits," IEEE Microwave Magazine, vol. 2, pp. 59-71, Dec. 2001.

[4] Z. Wang, B. D. Jensen, J. L. Volakis, K. Saitou, and K. Kurabayashi, "Analysis of RF-MEMS switches using finite element-boundary integration with moment method," in 2003 IEEE Antennas and Propagation Society International Symposium, 2003.

[5] R. Holm. Electric Contacts, Theory and Applications. Berlin, Germany: Spring-Verlag, 1976.

[6] P. G. Slade. Electrical Contacts: Principles and Applications. New York: Marcel Dekker, 1999. 